\title{
Noise mapping based on participative measurements with a smartphone
}

J. Picaut, P. Aumond, A. Can, N. Fortin, B. Gauvreau Environmental Acoustics Laboratory (fisttar), France

E. Bocher, S. Palominos, G. Petit Lab-STICC UMR CNRS 6285 (UBS), France

G. Guillaume

Acoustics unit (Cerema Est), France

ENERG GÉO̊PAL

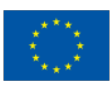




\section{Introduction}

- (Effect of) Noise in Europe:

"Excessive noise seriously harms human health and interferes with people's daily activities at school, at work, at home and during leisure time. It can disturb sleep, cause cardiovascular and psychophysiological effects, reduce performance and provoke annoyance responses and changes in social behaviour."

- European Environmental Noise directive 2002/49/EC "define a common approach intended to avoid, prevent or reduce on a prioritised basis the harmful effects, including annoyance, due to the exposure to environmental noise" 


\section{Introduction}

- Noise mapping:

- To identify noise pollution

- To propose action plans to reduce noise

- To communicate with citizens

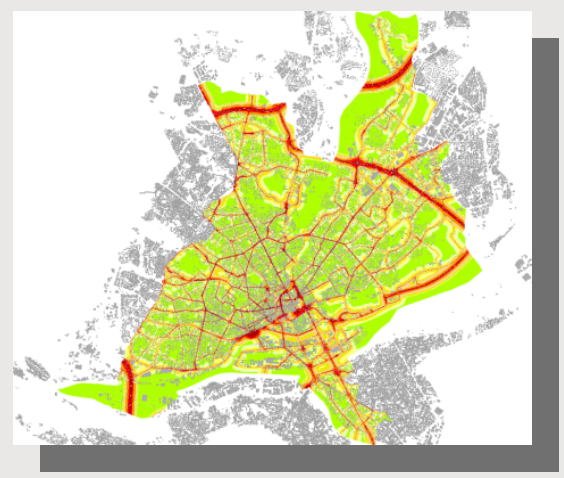

- Methods:

- Modelling of noise emission and propagation (NMPB, CNOSSOS, ISO 9613...)

- Measuring real noise in urban areas (noise observatories): need a large amount of "qualified" data. 


\section{Introduction}

- Alternative approach:

Develop a participative approach based on collaborative noise maps using smartphones (i.e. citizens) for a massive acquisition of noise data in urban environment

G. Guillaume et al, « Noise mapping based on participative measurements ».

Noise Mapping 3, no 1 (2016): 140-156. doi:10.1515/noise-2016-0011.

- Made by specialists in Geomatics and in Acoustics

- Citizen both producer and consumer of data

- Build a qualified noise database

- Produce "real" and relevant noise maps

- Share database and noise maps with communities 


\section{Summary}

- Introduction

- General overview

- NoiseCapture application

- Spatial Data Infrastructure (SDI)

- Processing data

- Conclusion \& prospects 


\section{General overview}

\section{NoiseCapture Android App}
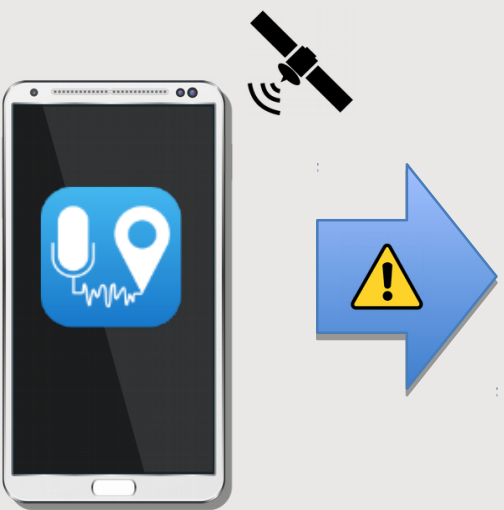

G. Guillaume et al, Noise Mapping 3 , no 1 (2016): 140156.

doi:10.1515/noise2016-0011.

\section{OnoMap}

SDI

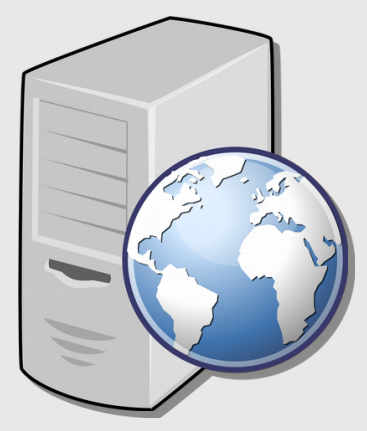

E. Bocher et al, OGRS 2016 Perugia, Italie: PeerJ Preprints, 2016. doi:https://doi.org/10.7 287/peerj.preprints. 22 73v2.

\section{Raw database}
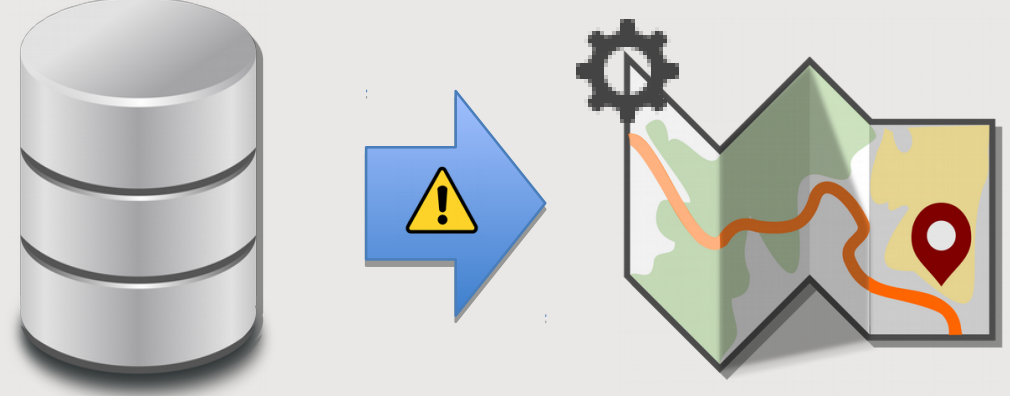

E. Bocher et al «Collaborative noise data collected from smartphones", Data in Brief (2017, to be published)

Noise maps
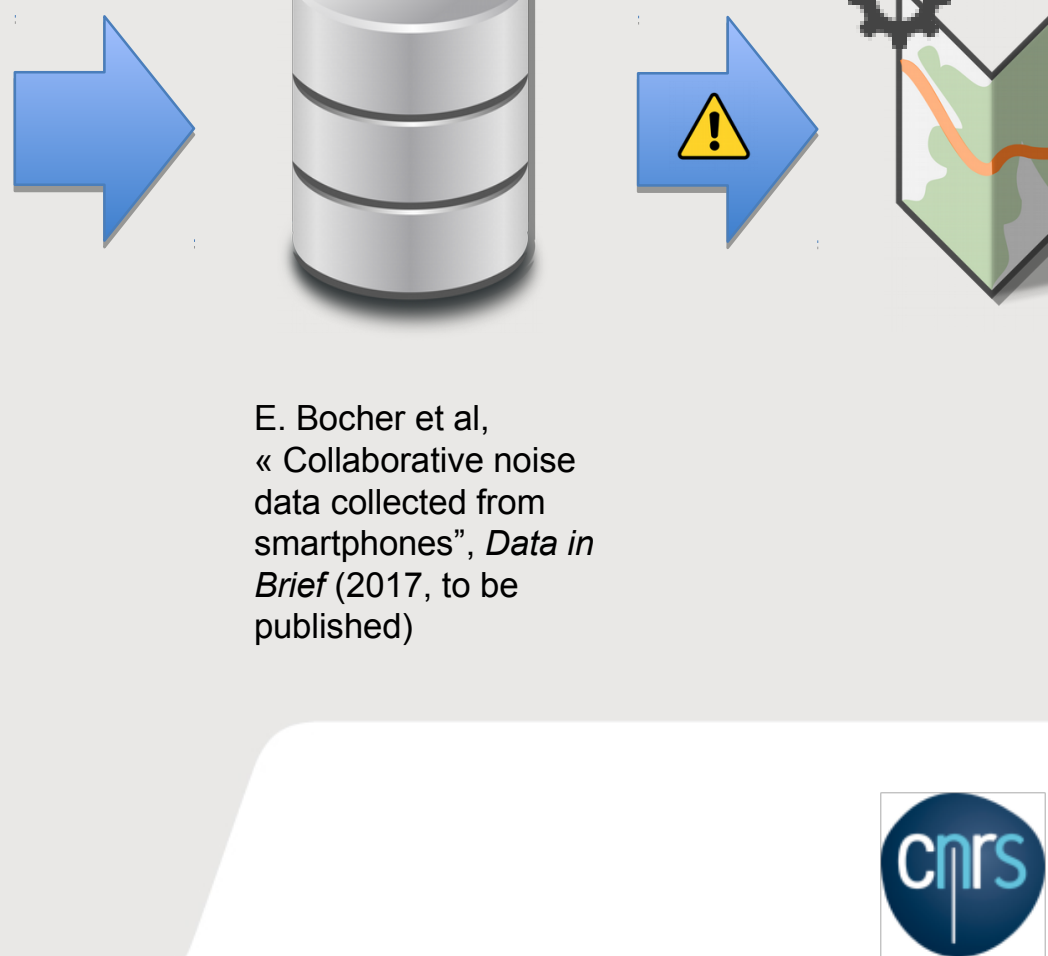


\section{NoiseCapture Android App}
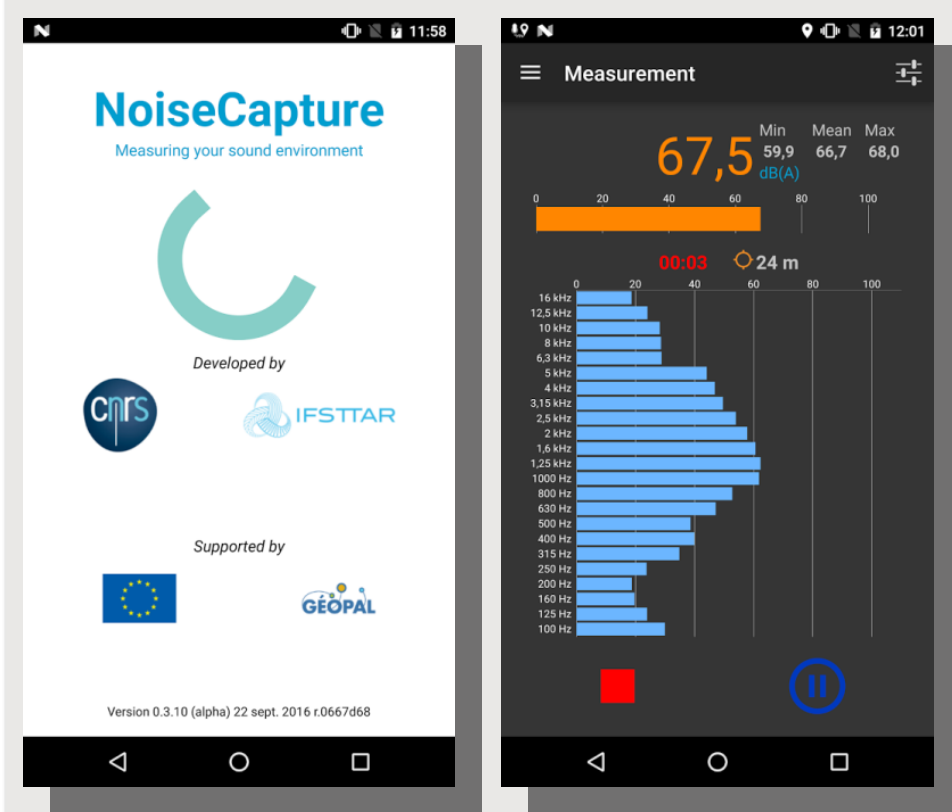

Measurement

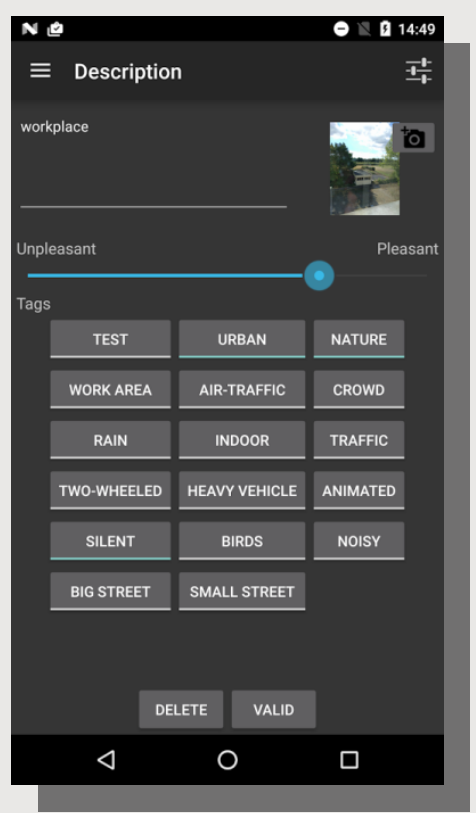

Description

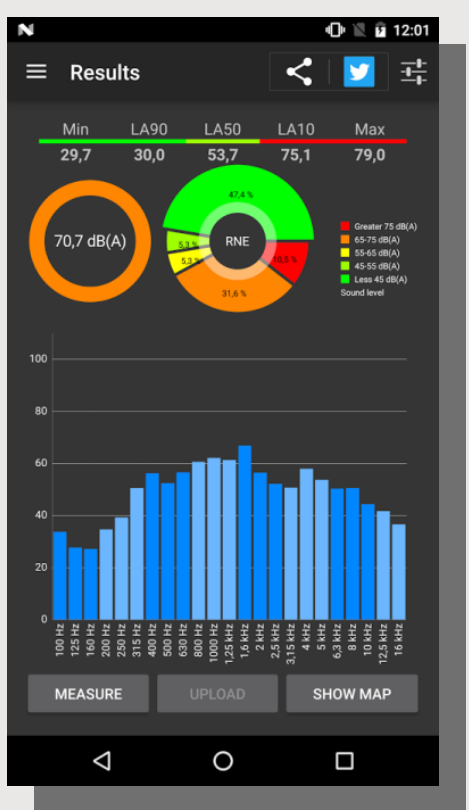

Indicators

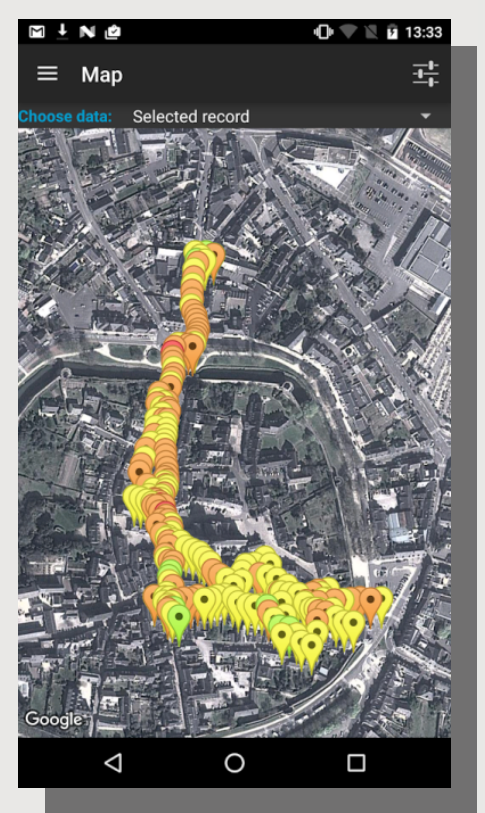

Visualization 


\section{NoiseCapture Android App}

- Audio restrictions (SHOULD / MUST since Android 4.3)

- Format:

- Linear PCM, 16-bit

- Sampling rates $(\mathrm{Hz})$ :

- 8000, 11025, 16000, (22500), 44100, (48000)

- Frequency response:

- $\pm 3 \mathrm{~dB}$, from $100 \mathrm{~Hz}$ to $4000 \mathrm{~Hz}$

- Amplitude response:

- $30 \mathrm{~dB}$ range from -18 dB to +12 dB @ 90 dB SPL

- Total harmonic distortion:

- less than $1 \%$ for $1000 \mathrm{~Hz}$ at $90 \mathrm{~dB}$ SPL 


\section{NoiseCapture Android App}

- Audio measurement

- Noise reduction processing disabled

- Automatic gain control disabled

- Respect of the audio input sensitivity 90dB@1000Hz

- Audio processing

- No audio recording (anonymised data, privacy policy)

- FFT analysis (short@1s \& fast@125ms)

- Calibration with a reference device

- Noise indicators calculation: LAeq, LA\%, spectrum 


\section{OnoMap SDI}

Metadata catalog

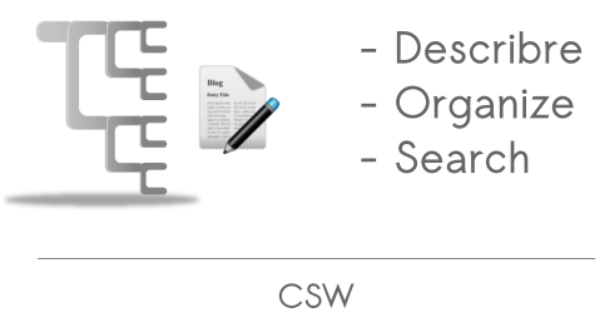

Server side
Spatial database

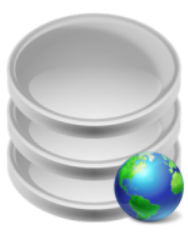

- Store

- Manage

- Query

\section{SFS SQL}

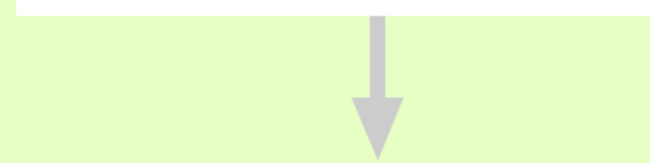

Cartographic server

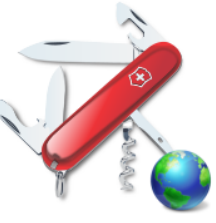

- Connect

- Style

- Transform

- Share

WMS / WFS
Processing

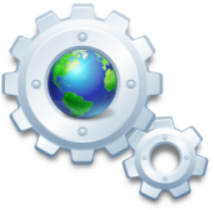

Process

data

remotely
WebCarto library

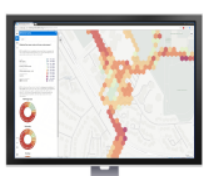

Display in an interactive way

E. Bocher et al. «OnoM@p : A Spatial Data Infrastructure Dedicated to Noise Monitoring Based on Volunteers Measurements ». In OGRS 2016, 11. Perugia, Italie: PeerJ Preprints, 2016.

doi:https://doi.org/10.7287/peerj.preprints.2273v2. 


\section{Raw database (online: http://data.noise-planet.org/noisecapture)}

\section{GeoJSON files}

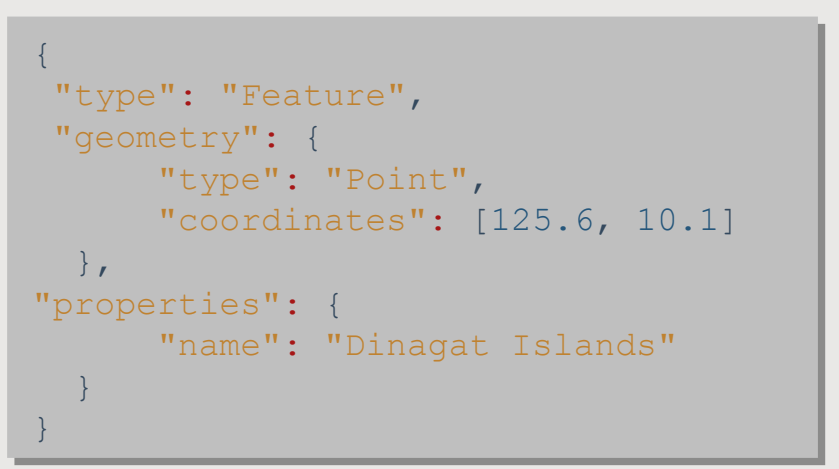

*.points.geojson

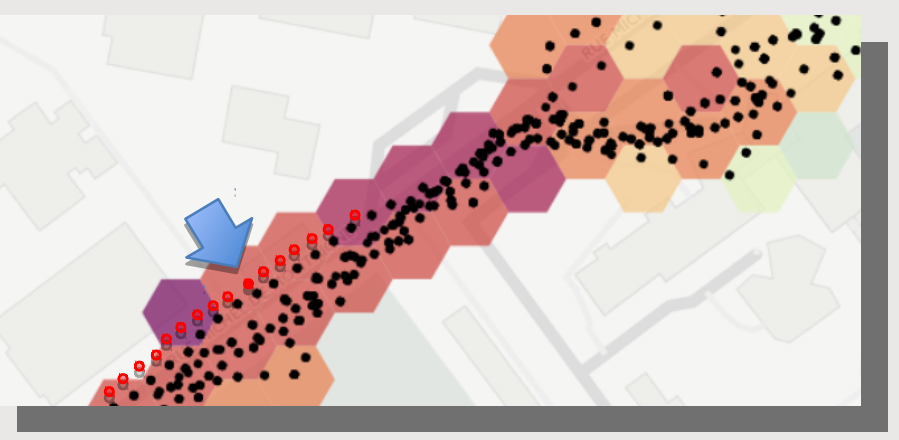

*.tracks.geojson

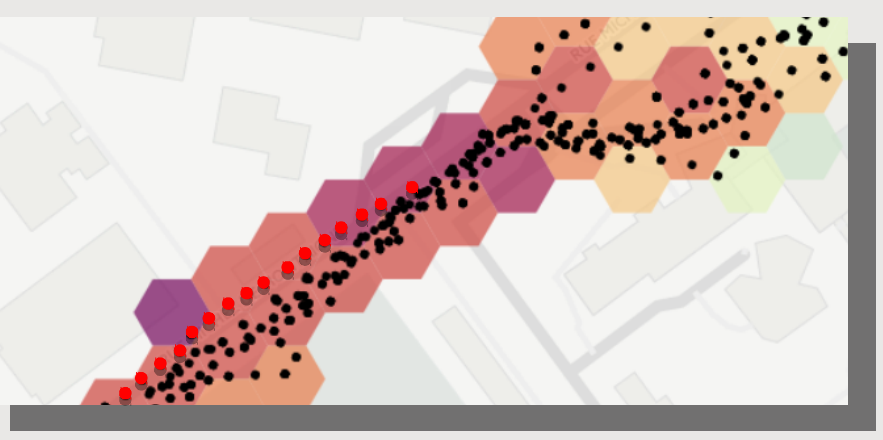

${ }^{*}$.areas.geojson

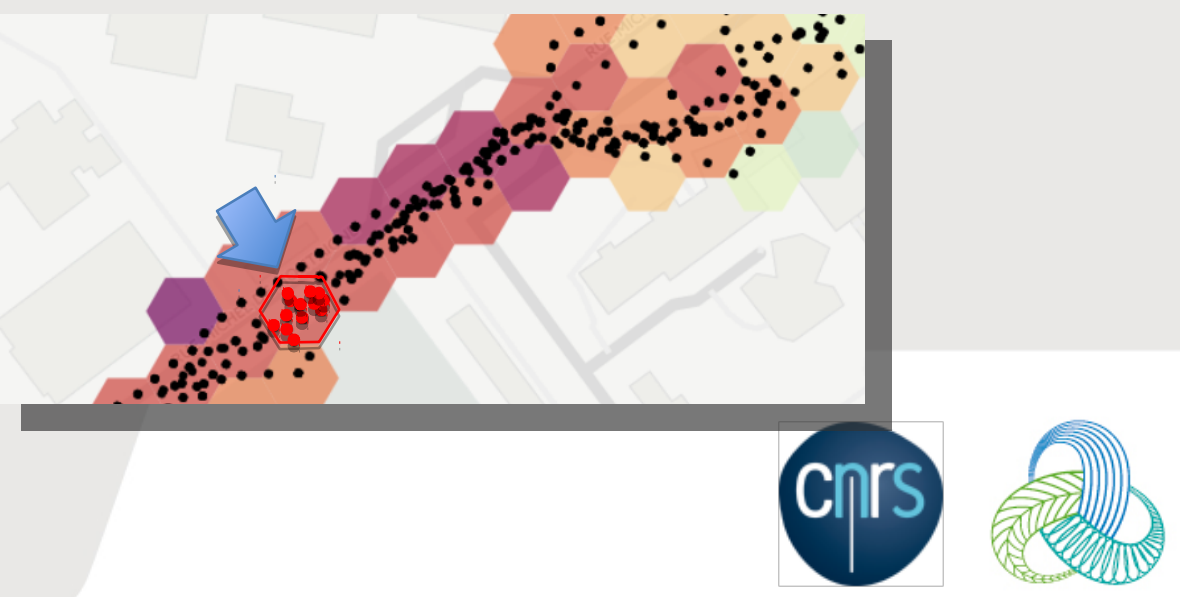




\section{Raw database}

\section{Be careful on the relevance of data}
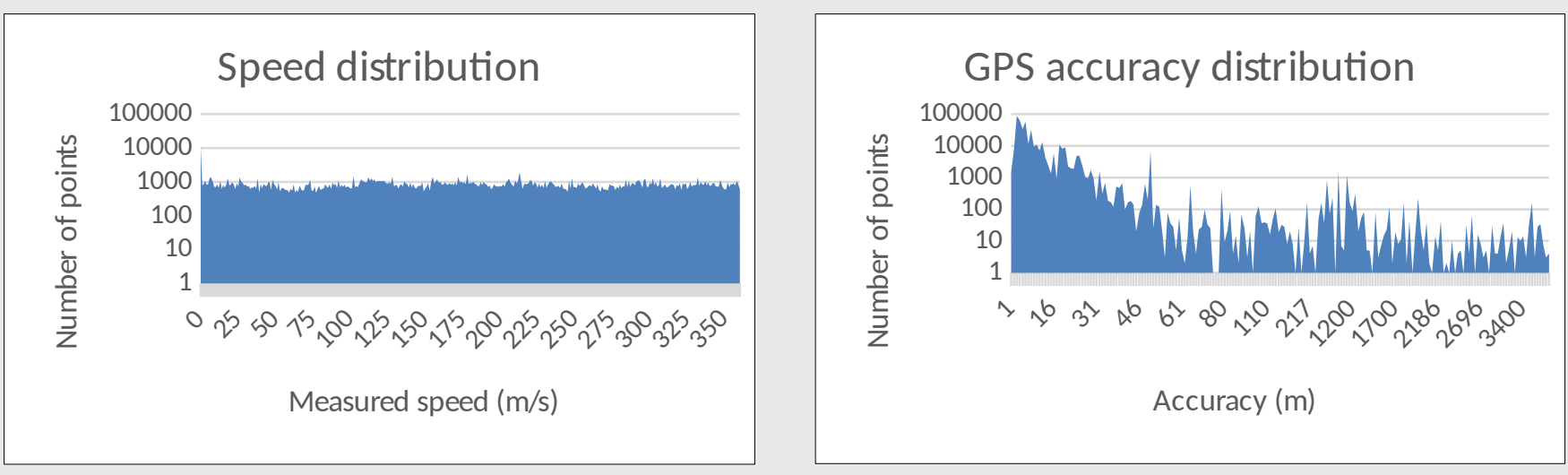

\section{Information provided by the user}
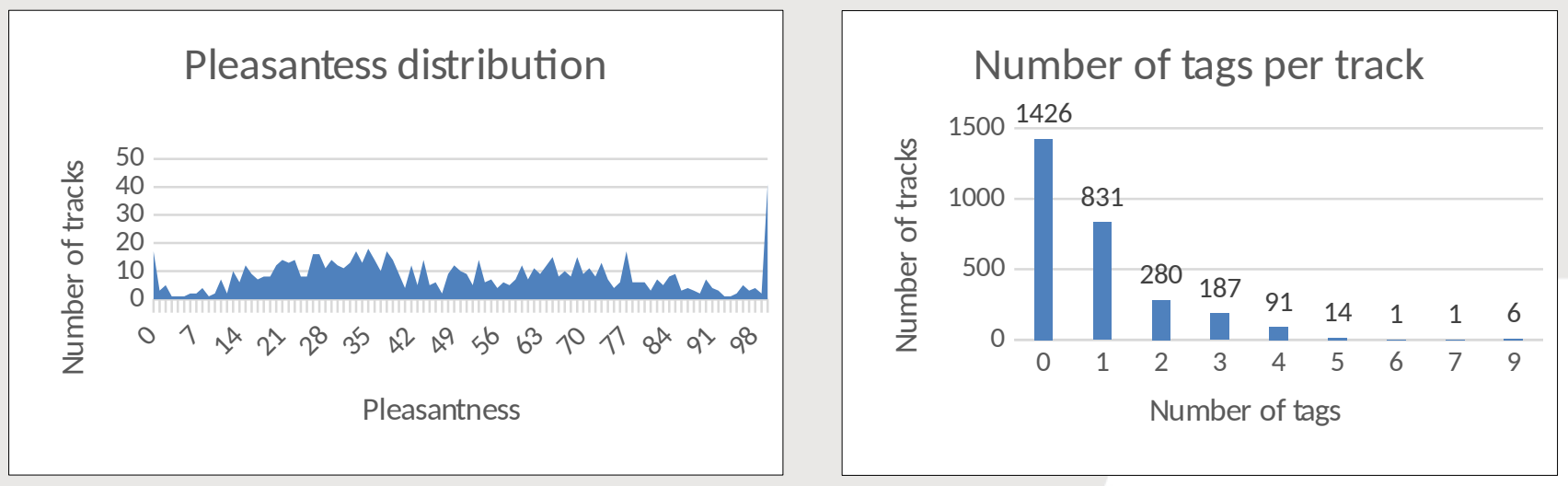

\section{Tracks \\ 2837}

Points 470869

Points with speed $116004_{(27 \%)}$

Tracks with pleasantness $1972(70 \%)$ Tracks with tags $1411_{(50 \%)}$ 


\section{Noise maps (first level: no filtering)}
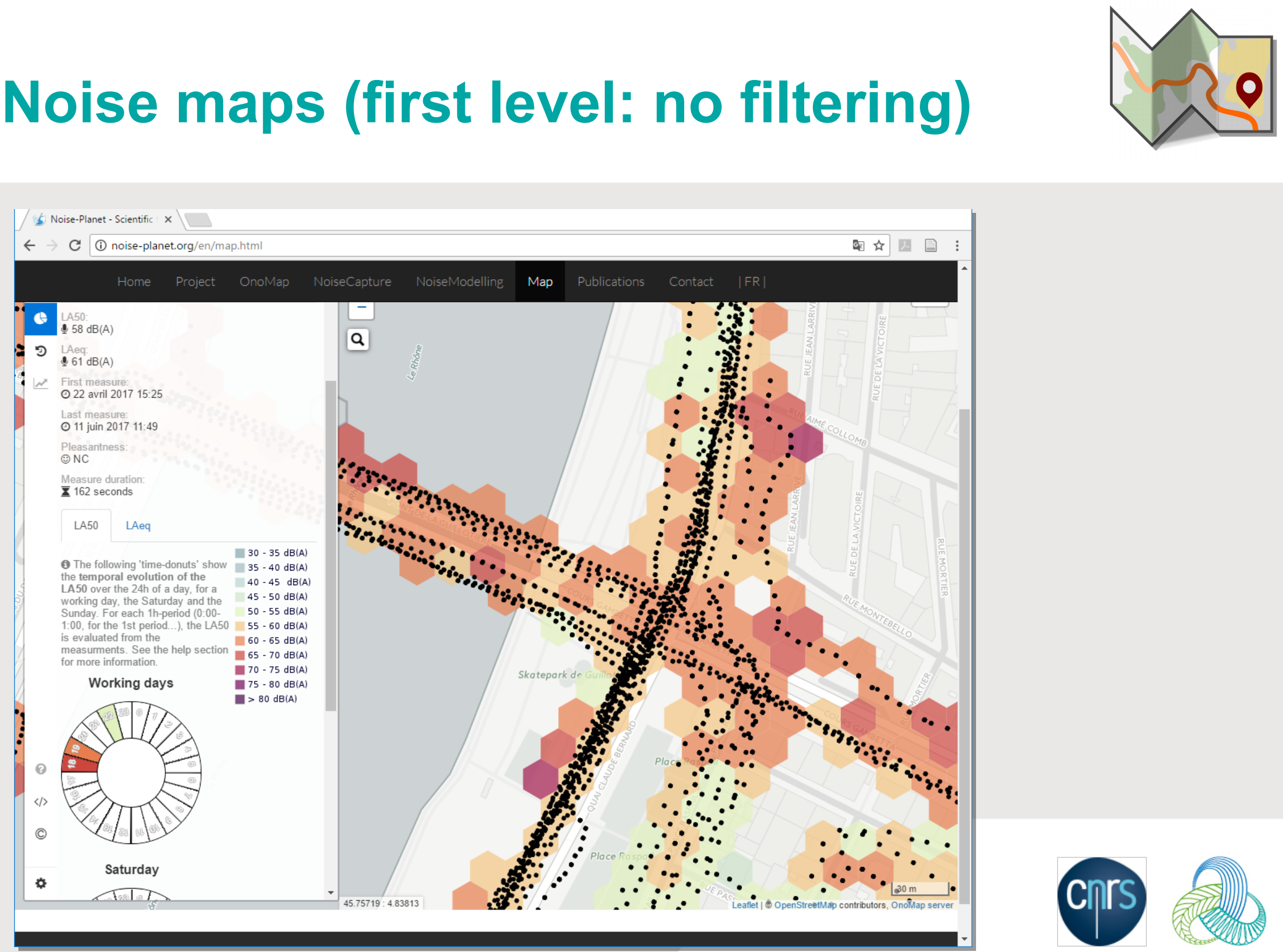


\section{Noise maps (second level)}

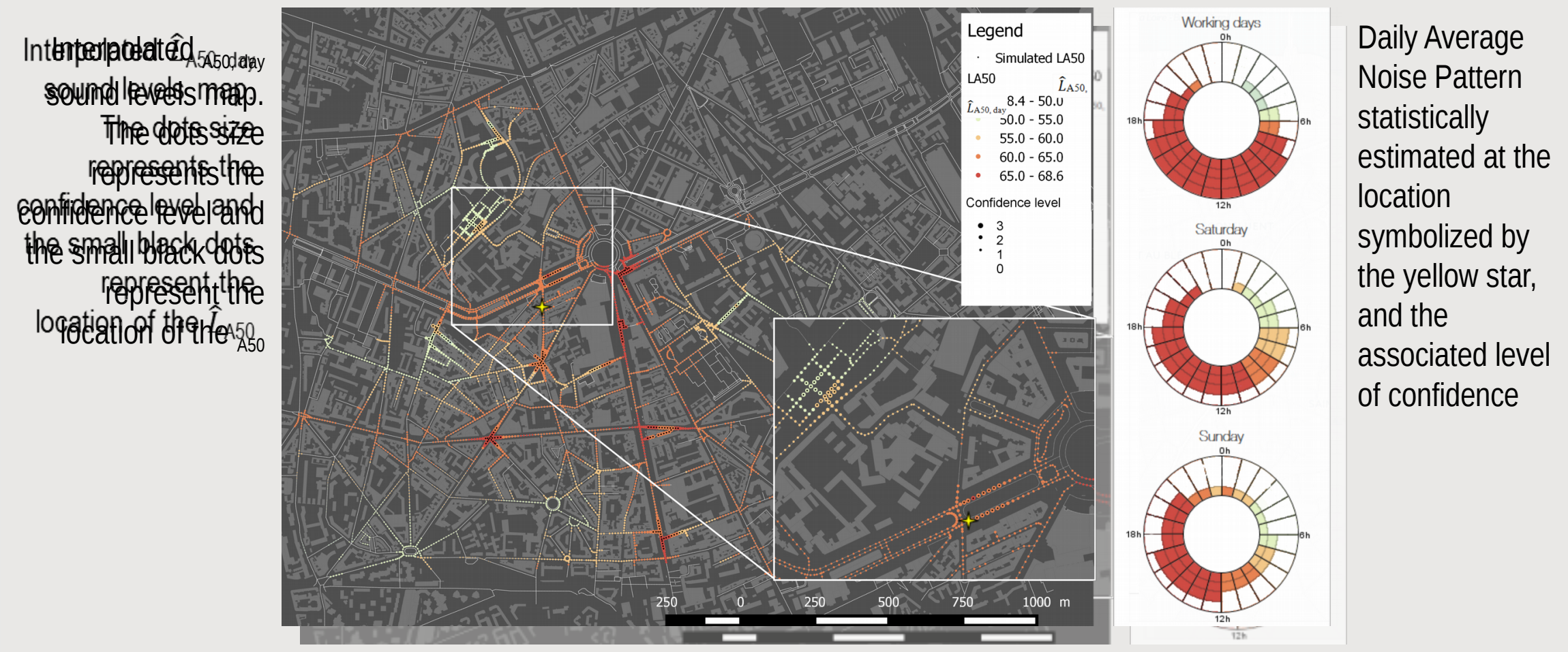

See P. Aumond et al, Acoustic mapping based on measurements: space and time interpolation, Internoise 2017, Hong Kong 


\section{Conclusion \& prospects}

$\checkmark$ A comprehensive and open infrastructure for producing noise data from smartphones, with a special attention (but perfectible) to the audio-processing

$\checkmark$ A raw database for the need of the scientific community in order to produce a relevant evaluation of noise in the environment

* Enhancement of the calibration and audio process

+ Methodologies for Data Quality Assessment

+ Production of noise maps for a more relevant assessment of the quality of the noise environment

+ Manage temporal vs spatial data 


\section{Thank you for your attention}

More information on the project:

http://noise-planet.org/en/index.html

Download NoiseCapture App on Google Play:

https://play.google.com/store/apps/details?id=org.noise_planet.noisecapture

Community noise maps:

http://noise-planet.org/en/map.html

Download data (per country):

http://data.noise-planet.org/en/index.html

Follow the development of NoiseCapture on GitHub plateform: https://github.com//fsttar/NoiseCapture

The EU ENERGIC-OD Project is funded under the ICT Policy Support Program (ICT PSP) as part of the Competitiveness and Innovation Framework Program of the European Community, and supported by the French geographic portal GÉOPAL of the Pays de la Loire region (France).

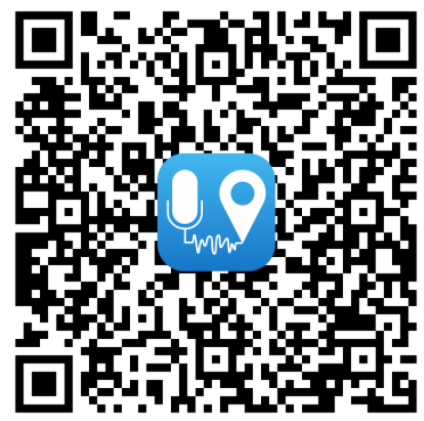

icaut - Acoustics'2017 Boston - Page 16 


\section{Raw database}

\section{${ }^{*}$.tracks.geojson (raw data)}

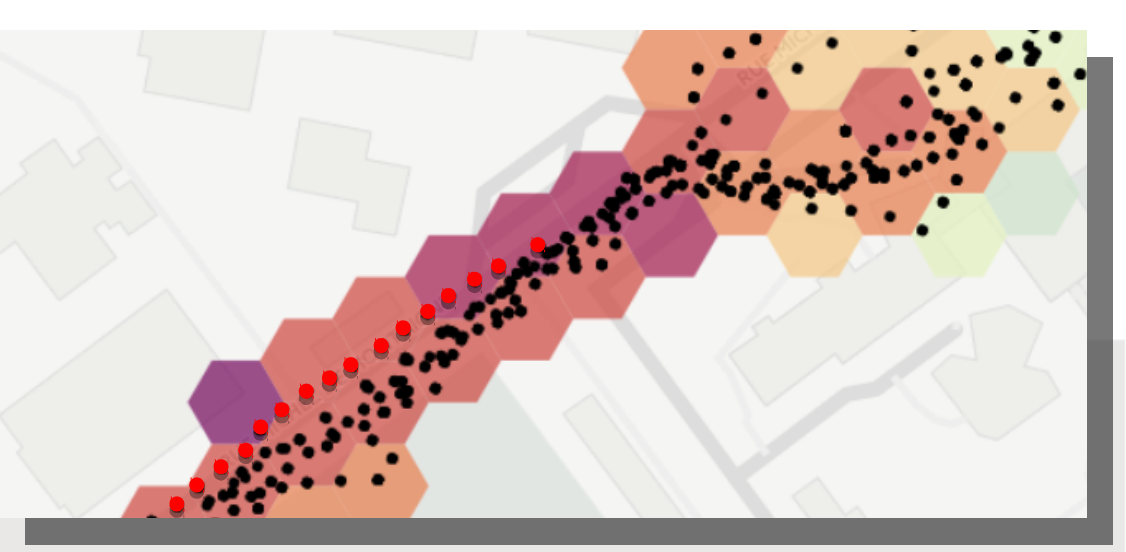

- time ISO8601: start time of measurement in ISO 8601

- time_epoch: start time of measurement in UTC

- pk track: database track primary key

- track_uuid: track universally unique identifier

- gain_calibration: signal gain calibration in dB

- noise level: LAeq along the track

- Tags: user supplied noise tags

- Pleasantness: user supplied pleasantness 0-100 


\section{Raw database}

*.points.geojson (raw data)

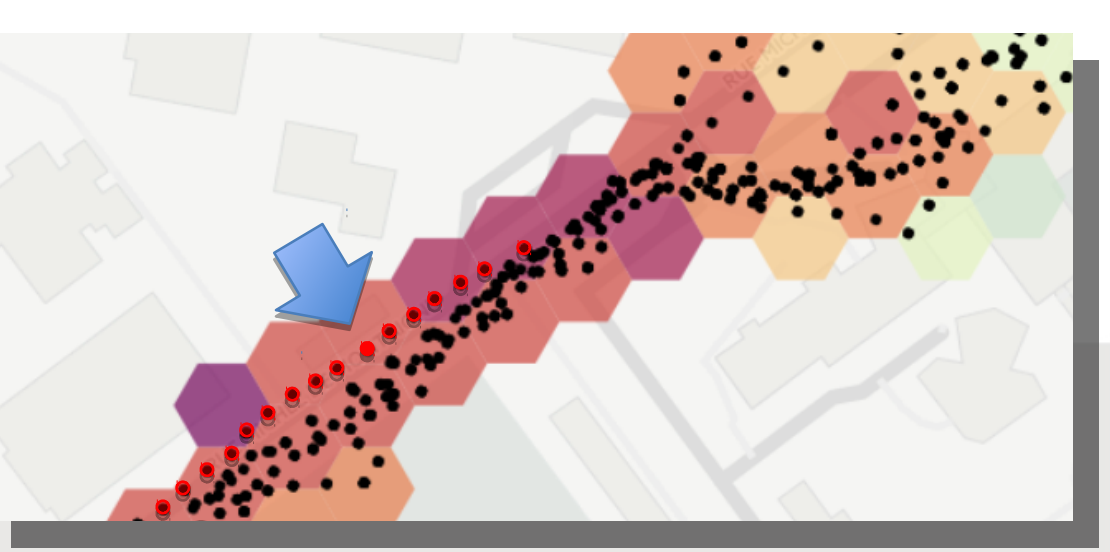

- pk_track: track primary key

- time ISO8601: measurement time in ISO 8601

- time epoch: measurement time in UTC

- time_gPs_IS08601: GPS measurement time in ISO 8601

- time_gps_epoch: GPS measurement time in UTC

- Noise_level: LAeq,1s in $\mathrm{dB}(\mathrm{A})$

- speed: GPS provided speed (not accurate)

- Orientation: GPS provided orientation (not accurate)

- Accuracy: GPS localization accuracy in meters 


\section{Raw database}

\section{${ }^{*}$.areas.geojson (pre-process)}

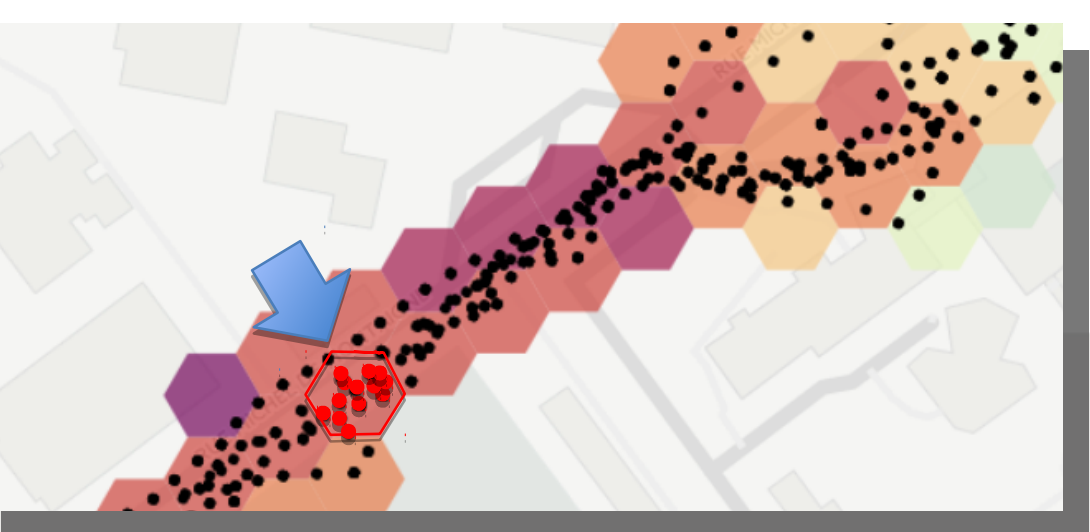

- cell_q: hexagon q coordinate (EPSG:3857-WGS84)

- cell_r: hexagon $r$ coordinate (EPSG:3857-WGS84)

- La50: LA50 in dB(A)

- laeq: LAeq in $\mathrm{dB}(\mathrm{A})$

- mean_pleasantness: mean pleasantness

- measure count: number of seconds of measurements

- first measure ISO 8601: date of the first measurement

- first_measure_epoch: date of the first measurement

- last measure ISO 8601: date of the last measurement

- last measure epoch: date of the last measurement

- leq_profile: 72 hours LAeq levels 